\title{
Capsule Commentary on Rivera-Caravaca et al., Soluble Fibrin Monomer Complex and Prediction of Cardiovascular Events in Atrial Fibrillation: The Observational Murcia Atrial Fibrillation Project
}

\author{
Bethany T. Samuelson Bannow, MD \\ University of Washington, Seattle, WA, USA. \\ J Gen Intern Med 33(6):945 \\ DOI: $10.1007 / \mathrm{s} 11606-018-4320-2$ \\ (c) Society of General Internal Medicine 2018
}

$\mathrm{R}$ ivera-Caravaca et al. ${ }^{1}$ report clinical outcomes in association with varying levels of soluble fibrin monomer complex (SFMC) in 1226 patients with atrial fibrillation receiving vitamin $\mathrm{K}$ antagonists (VKAs) for stroke prevention. The outcomes examined were highly clinically relevant and included ischemic stroke, a composite of adverse cardiovascular events including mortality, cardiovascular mortality and all-cause mortality. While associations between high SFMC levels $(>12 \mu \mathrm{g} / \mathrm{mL})$ and cardiovascular event and mortality outcomes were noted on multivariate analysis, no association with ischemic stroke was found.

The $\mathrm{CHA}_{2} \mathrm{DS}_{2}$-VASc score, which utilizes seven clinical features to predict risk of ischemic stroke among patients with atrial fibrillation, is a well-established risk prediction model used to inform decision making regarding prophylactic anticoagulation in such patients. ${ }^{2}$ Acknowledging this, the authors appropriately investigated whether a finding of elevated SFMC levels impacted the clinical usefulness of this prediction tool. No significant improvement was found.

This article is an excellent example of a well-planned and executed but early investigation of a biomarker. As such, caution is required in interpreting the clinical relevance of these findings. The most important limitation of this study is that all patients were already on anticoagulation with VKAs. As previously stated, the purpose of risk prediction models in the clinical care of patients with atrial fibrillation is generally to inform decisions regarding the initiation of anticoagulation. Furthermore, such decisions are generally based upon risk of

ischemic stroke - the one outcome for which this marker lacked any predictive value - and in many practices, the use of VKAs is being phased out in favor of newer, safer direct oral anticoagulants.

As the authors state, this assay does not add significantly to the clinical utility of existing risk prediction models, and certainly at this point, it would be premature to base any clinical decisions upon these findings. However, the promising findings of associations between high SFMC levels and important cardiovascular and mortality outcomes suggest that further studies, ideally in patients who have not yet started anticoagulation, may demonstrate clinical utility. The astute clinician will eagerly anticipate the results of such studies as they may yet be practice-changing.

Corresponding Author: Bethany T. Samuelson Bannow, MD; University of Washington, Seattle, WA, USA (e-mail: Bts99@uw.edu).

\section{Compliance with Ethical Standards:}

Conflict of Interest: The author has no conflicts of interest with this article.

\section{REFERENCES}

1. Rivera-Caravaca JM, Roldán V, Romera M, Esteve-Pastor MA, Valdés M, Lip GYH, Vicente V, Marin F. Soluble fibrin monomer complex and prediction of cardiovascular events in atrial fibrillation: the observational Murcia atrial fibrillation project. J Gen Intern Med. https://doi.org/10. 1007/s11606-017-4279-4

2. Lip GY, Nieuwlaat R, Pisters R, Lane DA, Crijns HJ. Refining clinical risk stratification for predicting stroke and thromboembolism in atrial fibrillation using a novel risk factor-based approach: the euro heart survey on atrial fibrillation. Chest. 2010;137:263-72. https://doi.org/10.1378/ chest.09-1584.

Published online January 29, 2018 